\title{
Betweenness Centrality of Some Complementary Prism Graphs
}

\author{
Aysun AYTAÇ*1, Canan ÇiFTÇí ${ }^{2}$ \\ ${ }^{1}$ Ege University, Faculty of Science, Department of Mathematics, 35100, İzmir \\ (ORCID:https://orcid.org/0000-0003-2086-8969) \\ ${ }^{2}$ Ordu University, Faculty of Arts and Sciences, Department of Mathematics, 52200, Ordu \\ (ORCID:https://orcid.org/0000-0001-5397-0367)
}

(Alınıs / Received: 02.07.2018, Kabul / Accepted: 02.05.2019, Online Yayınlanma / Published Online: 30.08.2019)

Keywords
Graph theory,
Network design and
communication,
Betweenness centrality,
Complementary prism graph

\begin{abstract}
There are a lot of centrality measures that have been introduced for networks. One of them is betweenness centrality. It is a measure of the influence of a vertex over the flow of information between all pairs of vertices. This information flows over the shortest paths between these vertices. The fact that any vertex has a high value of centrality indicates that what level this vertex is in connection with vertices which are not adjacent with each other. Since this vertex controls flows of information, it has a potential role in the network. In this paper, we study on the betweenness centrality of some complementary prism graphs.
\end{abstract}

\section{Bazı Tümleyen Prizma Grafların Arasındalık Merkezliği}

\section{Anahtar Kelimeler}

Graf teori,

A $\breve{g}$ tasarımı ve iletişim, Arasındalık merkezliği, Tümleyen prizma graf

\begin{abstract}
Özet: Literatürde ağlar için tanımlanmış birçok merkezlik ölçümü vardır. Bunlardan biri arasındalık merkezliğidir. Arasındalık merkezliği bir tepenin tüm tepe çiftleri arasındaki bilgi akışına etkisinin bir ölçümüdür. Bu bilgi akışı, tepeler arasındaki en kısa yollar üzerinde olmaktadır. Herhangi bir tepenin yüksek arasındalık merkezliğe sahip olması o tepenin birbiriyle komşu olmayan tepelerle ne düzeyde bağlantı içinde olduğunu göstermektedir. Bu tepe ağdaki bilgi akışını kontrol ettiğinden ağda önemli bir yere sahiptir. Bu makalede bazı tümleyen prizma grafların arasındalık merkezliği üzerine çalışılmıştır.
\end{abstract}

\section{Introduction}

There are a lot of important properties for a network. One of them is which vertices lie on the shortest paths (geodesics) among pairs of other vertices [1,2]. Betweenness centrality is based on shortest paths enumeration. It determines the importance or the centrality of a vertex (or an edge) in a network and plays an important role in analysis of social or communication networks [3], computer networks [4] and many other types of network data models $[5,6]$. For example, in a telecommunication network, vertices with the higher value of centrality are more important. Because, more information passes through these vertices than the others. Since they lie on the largest number of paths taken by messages, removing these vertices from the network cuts off communications between others. Hence, the betweenness centrality is related to a network's connectivity and therefore its reliability [7].

The concept of betweenness centrality was first introduced by Bavelas [8] in 1948. Particularly, this concept is used in human communication in this study and and it indicates that when a person in a group is located on the shortest communication path connecting pairs of others, that person is in central position [9].

Betweenness centrality $C_{B}(v)$ for a vertex $v$ is defined as

$$
C_{B}(v)=\sum_{s \neq v \neq t} \frac{\sigma_{s t}(v)}{\sigma_{s t}},
$$

where $\sigma_{s t}$ is the number of shortest paths with vertices $s$ and $t$ as their end vertices, while $\sigma_{s t}(v)$ is the number of those shortest paths that include vertex $v$.

The betweenness centrality of a graph $G$ on $n$ vertices is defined as

$$
C_{B}(G)=\frac{2 \sum_{i=1}^{n}\left[C_{B}\left(v^{*}\right)-C_{B}\left(v_{i}\right)\right]}{(n-1)^{2}(n-2)},
$$

where $C_{B}\left(v^{*}\right)$ is the largest value of $C_{B}\left(v_{i}\right)$ for any vertex $v_{i}$ in the given graph $G$.

This paper determines betweenness centrality of some complementary prism graphs. In 2007, Haynes et al. in [10] introduced the complementary product as a generalization of the Cartesian product. Complementary prisms of a graph 


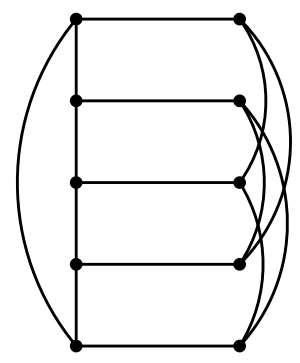

$C_{5} \bar{C}_{5}$

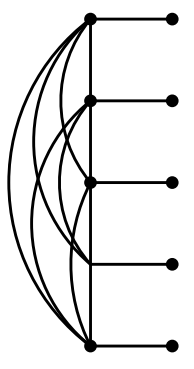

$K_{5} \bar{K}_{5}$
Figure 1. The Petersen graph $C_{5} \bar{C}_{5}$ and the corona $K_{5}$ 。 $K_{1}$

$G$ is the subset of complementary products. Let $G$ be a graph and $\bar{G}$ be the complement of $G$. The complementary prism $G \bar{G}$ of $G$ is the graph formed from the disjoint union $G \cup \bar{G}$ of $G$ and $\bar{G}$ by adding the edges of a perfect matching between the corresponding vertices of $G$ and $\bar{G}$. In other words, for a graph $G$ with vertex set $V(G)$ and edge set $E(G)$ the complementary prism of $G$ is the graph with vertex set $V(G \bar{G})=\left\{v_{1}, v_{2}, \ldots, v_{n}\right\} \cup\left\{\bar{v}_{1}, \bar{v}_{2}, \ldots, \bar{v}_{n}\right\}$ and edge set

$$
\begin{aligned}
E(G \bar{G})= & E(G) \cup\left\{\bar{v}_{i} \bar{v}_{j}: 1 \leqslant i<j \leqslant n \quad \text { and } \quad v_{i} v_{j} \notin E(G)\right\} \\
& \cup\left\{v_{1} \bar{v}_{1}, v_{2} \bar{v}_{2}, \ldots, v_{n} \bar{v}_{n}\right\},
\end{aligned}
$$

in which for a vertex $v$ of $G$, vertex $\bar{v}$ is the corresponding vertex in $\bar{G}[11,12]$. As demonstrated in Figure 1, the graph $C_{5} \bar{C}_{5}$ is the Petersen graph. Also, the graph $K_{n} \bar{K}_{n}$ is the corona $K_{n} \circ K_{1}$, where the corona $K_{n} \circ K_{1}$ is the graph obtained from $K_{n}$ by attaching a pendant edge to each vertex of $K_{n}$. Complementary prisms are investigated in [11-15].

For notation and graph theory terminology we in general follow [16]. Before stating our results, we give some notations and formal definitions. Let $G=(V(G), E(G))$ be a graph with vertex set $V(G)$ and edge set $E(G)$. The $o r$ der of $G$ is the number of vertices of $G$. Given any two vertices $u, v \in V(G)$, the distance $d(u, v)$ is the length of the shortest path or geodesic path between $u$ and $v$. The diameter $\operatorname{diam}(G)$ of a graph $G$ is the maximum distance between two vertices of $G$. The degree of a vertex $v$ in a graph $G$ is the number of edges of $G$ incident to $v$ and denoted by $\operatorname{deg}_{G}(v)$. Throughout this paper, $\operatorname{deg}(v)$ represents $\operatorname{deg}_{G \bar{G}}(v)$ for any vertex $v$ in $G \bar{G}$. The center vertex of a star or wheel graph is the only vertex that has a maximum degree.

\section{Betweenness Centrality of Some Complementary Prism Graphs}

In this section, we first state two known theorems that we use in the proof of our results. Next, we determine the betweenness centrality of some complementary prism graphs.
Theorem 2.1. [2] The betweenness centrality of a vertex $v$ in $S_{n}$ is given by

$$
C_{B}(v)= \begin{cases}\left(\begin{array}{c}
n-1 \\
2
\end{array}\right), & \text { for center vertex } \\
0, & \text { for other vertices }\end{cases}
$$

Theorem 2.2. [2] The betweenness centrality of a vertex $v$ in a wheel graph $W_{n}, n>5$ is given by

$$
C_{B}(v)= \begin{cases}\frac{(n-1)(n-5)}{2}, & \text { if } v \text { is center vertex } \\ \frac{1}{2}, & \text { otherwise }\end{cases}
$$

Theorem 2.3. Let $K_{n} \bar{K}_{n}$ be the complementary prism of a complete graph on $2 n$ vertices. Then the betweenness centrality of a vertex $v$ in $K_{n} \bar{K}_{n}$ is given by

$$
C_{B}(v)= \begin{cases}2 n-2, & \text { if } v \text { is in } K_{n}, \\ 0, & \text { otherwise. }\end{cases}
$$

Proof. Take a vertex $v$ in $K_{n}$. On $K_{n}$, there exists $n-1$ adjacent vertices of $v$ and each pair of these vertices contributes 0 to $v$. Consider any adjacent vertex of $v$ in $K_{n}$. There is only one geodesic path joining this vertex to corresponding vertex of $v$ and it passes through $v$. Thus, each pair contributes centrality 1 to $v$ and gives a total of $n-1$. Now, consider any vertex in $\bar{K}_{n}$ other than corresponding vertex of $v$. There is only one geodesic path from this vertex to corresponding vertex of $v$ passing through $v$, and it contributes a betweenness centrality 1 to $v$. Since there are $n-1$ such pairs, they provide a betweenness centrality $n-1$ to $v$. Hence, the betweenness centrality of any vertex $v$ in $K_{n}$ is $2 n-2$.

Take a vertex $\bar{v}$ in $\bar{K}_{n}$. Since $\operatorname{deg}(\bar{v})=1$, there is one path joining vertex $\bar{v}$ and all other vertices of $K_{n} \bar{K}_{n}$. However, it does not pass through $\bar{v}$. Then the betweenness centrality of $\bar{v}$ is 0 .

Maximum value of the betweenness centrality of vertices in $K_{n} \bar{K}_{n}$ and the graph centrality are as follows:

$$
\begin{gathered}
C_{B}\left(v^{*}\right)=2 n-2, \\
C_{B}\left(K_{n} \bar{K}_{n}\right)=\frac{2 \sum_{i=1}^{2 n}\left[C_{B}\left(v^{*}\right)-C_{B}\left(v_{i}\right)\right]}{(2 n-1)^{2}(2 n-2)}=\frac{2 n}{(2 n-1)^{2}} .
\end{gathered}
$$

Theorem 2.4. Let $S_{n} \bar{S}_{n}$ be the complementary prism of a star on $2 n$ vertices and $c$ be the center vertex of $S_{n}$. Then the betweenness centrality of a vertex $v$ in $S_{n} \bar{S}_{n}$ is given by

$$
C_{B}(v)= \begin{cases}\frac{(n-1)(n+2)}{2}, & \text { for center vertex } c, \\ 0, & \text { for } \bar{c}, \\ 2, & \text { for any vertex in } S_{n}-\{c\}, \\ n-2, & \text { for any vertex in } \bar{S}_{n}-\{\bar{c}\},\end{cases}
$$

in which $\bar{c}$ is the corresponding vertex of $c$.

Proof. Let $v_{1}$ be the center vertex of $S_{n}$ in $S_{n} \bar{S}_{n}$. By Theorem 2.1, pairs of vertices in $S_{n}$ contribute $\left(\begin{array}{c}n-1 \\ 2\end{array}\right)$ to $v_{1}$. Consider the pairs $\left(\bar{v}_{1}, v_{i}\right)$ and $\left(\bar{v}_{1}, \bar{v}_{i}\right)$ for all 
$i \in\{2,3, \ldots, n\}$. Each pair has a geodesic path which passes through $v_{1}$ and contributes 1 to the centrality of $v_{1}$. Since there are $2 n-2$ such pairs, they give a total of $2 n-2$. Thus, the betweenness centrality of $v_{1}$ is $\left(\begin{array}{c}n-1 \\ 2\end{array}\right)+2 n-2=\frac{(n-1)(n+2)}{2}$.

Let $\bar{v}_{1}$ be the corresponding vertex of center vertex in $\bar{S}_{n}$. Since $\operatorname{deg}\left(\bar{v}_{1}\right)=1$, there are no pairs in $S_{n} \bar{S}_{n}$ which pass through $\bar{v}_{1}$. Thus, the betweenness centrality of $\bar{v}_{1}$ is 0 .

Consider any vertex $v_{i}$ in $S_{n}$, where $i \in\{2,3, \ldots, n\}$. None of $\left(\begin{array}{c}n-1 \\ 2\end{array}\right)$ pairs in $S_{n}$ contains $v_{i}$. However, there is a geodesic path joining corresponding vertex $\bar{v}_{i}$ and $v_{1}$ and $\bar{v}_{1}$ passing through $v_{i}$. Since each one contributes 1 to the centrality of $v_{i}$, the betweenness centrality of $v_{i}$ is 2 .

For any vertex $\bar{v}_{i}$, there exists $n-2$ adjacent vertices of $\bar{v}_{i}$ in $\bar{S}_{n}$, in which $i \in\{2,3, \ldots, n\}$. Then there is a geodesic path joining each adjacent vertex to $v_{i}$ and it passes through $\bar{v}_{i}$. Thus, each pair contributes centrality 1 to $\bar{v}_{i}$ and they contribute a total of $n-2$.

The largest value of the betweenness centrality of vertices of $S_{n} \bar{S}_{n}$ is

$C_{B}\left(v^{*}\right)=\frac{(n-1)(n+2)}{2}$,

and the betweenness centrality of $S_{n} \bar{S}_{n}$ is

$$
\begin{aligned}
C_{B}\left(S_{n} \bar{S}_{n}\right)= & \frac{2 \sum_{i=1}^{2 n}\left[C_{B}\left(v^{*}\right)-C_{B}\left(v_{i}\right)\right]}{(2 n-1)^{2}(2 n-2)}\left\{\left(\frac{(n-1)(n+2)}{2}-0\right)\right. \\
= & \frac{2}{(2 n-1)^{2}(2 n-2)} \\
& +(n-1)\left(\frac{(n-1)(n+2)}{2}-2\right) \\
& \left.+(n-1)\left(\frac{(n-1)(n+2)}{2}-(n-2)\right)\right\} \\
= & \frac{2 n^{2}+n-2}{2(2 n-1)^{2}} .
\end{aligned}
$$

Theorem 2.5. Let $P_{n} \bar{P}_{n}$ be the complementary prism of a path of order $2 n$. Then the betweenness centrality of vertices of $P_{n} \bar{P}_{n}$ for $n>6$ is given as follows:

If $v_{i} \in V\left(P_{n}\right)$, then

$$
C_{B}\left(v_{i}\right)= \begin{cases}1, & \text { if } i \in\{1, n\}, \\ \frac{7}{2}, & \text { if } i \in\{2, n-1\} \\ 4, & \text { if } i \in\{3,4, \ldots, n-2\}\end{cases}
$$

If $\bar{v}_{i} \in V\left(\bar{P}_{n}\right)$, then

$$
C_{B}\left(\bar{v}_{i}\right)= \begin{cases}2 n+\frac{1}{n-3}-\frac{9}{2}, & \text { if } i \in\{1, n\}, \\ 2 n+\frac{1}{n-3}+\frac{n-5}{n-4}-\frac{15}{2}, & \text { if } i \in\{2, n-1\}, \\ 2 n+\frac{1}{n-3}+\frac{n-6}{n-4}-\frac{17}{2}, & \text { if } i \in\{3, n-2\}, \\ 2 n+\frac{2}{n-3}+\frac{n-7}{n-4}-9, & \text { if } i \in\{4,5, \ldots, n-3\} .\end{cases}
$$

Proof. Since $\operatorname{diam}\left(P_{n} \bar{P}_{n}\right)=3$, there is a geodesic path of length at most 3 between two vertices in $P_{n} \bar{P}_{n}$. For the betweenness centrality of any vertex $v$ in $P_{n} \bar{P}_{n}$, we have two cases: when $v \in V\left(P_{n}\right)$ and when $v \in V\left(\bar{P}_{n}\right)$.

Case 1. Let $v_{i}$ be a vertex in $P_{n}$, where $i \in\{1,2, \ldots, n\}$. Since the distance between any non-adjacent vertices of $\bar{P}_{n}$ is 2, none of $\left(\begin{array}{l}n \\ 2\end{array}\right)$ pairs of vertices of $\bar{P}_{n}$ passes through $v_{i}$.

For all $i \geqslant 1$, consider the pair $\left(v_{j}, \bar{v}_{k}\right)$, where $j \in\{1,2, \ldots, n\}, j \neq i$ and $k \in\{1,2, \ldots, n\}$. There is a geodesic path joining $\bar{v}_{i}$ to adjacent vertices of $v_{i}$ in $P_{n}$ which passes through $v_{i}$. Since there are $\operatorname{deg}\left(v_{i}\right)-1$ adjacent vertices of $v_{i}$ in $P_{n}$, they contribute a betweenness centrality $\operatorname{deg}\left(v_{i}\right)-1$ to $v_{i}$.

For contribution of any pair of vertices in $P_{n}$ to the betweenness centrality of $v_{i}$, we partitioned the vertex set of $P_{n}$ into the following three pairs.

- Let $i \in\{1, n\}$.

Since the distance between any two vertices of $P_{n}$ is at most 3 in $P_{n} \bar{P}_{n}$, none of geodesic paths between these vertices except $v_{1}$ and $v_{n}$ contains $v_{1}$ or $v_{n}$. Thus, these pairs contribute to $v_{1}$ or $v_{n}$ the betweenness centrality zero.

As a consequence, we have

$$
C_{B}\left(v_{i}\right)=\operatorname{deg}\left(v_{i}\right)-1=1 \quad \text { for } \quad i \in\{1, n\},
$$

in which $\operatorname{deg}\left(v_{i}\right)=2$.

$$
\text { - Let } i \in\{2, n-1\} \text {. }
$$

For the pair $\left(v_{i-1}, v_{i+1}\right)$, there is a geodesic path of length 2 passing through $v_{i}$ and it contributes a betweenness centrality 1 to $v_{i}$. Moreover, consider the paths of length 3 containing $v_{i}$. There are two geodesic paths joining $v_{1}$ and $v_{4}$, one of which passes through $v_{2}$. Similarly, there are two geodesic paths joining $v_{n}$ and $v_{n-3}$, one of which passes through $v_{n-1}$. Hence, each pair contributes $\frac{1}{2}$ to $v_{i}$.

As a consequence, we have

$$
C_{B}\left(v_{i}\right)=\operatorname{deg}\left(v_{i}\right)-1+1+\frac{1}{2}=\frac{7}{2} \quad \text { for } \quad i \in\{2, n-1\},
$$

in which $\operatorname{deg}\left(v_{i}\right)=3$.

$$
\text { - Let } i \in\{3,4,5, \ldots, n-2\} \text {. }
$$

There is a geodesic path of length 2 between adjacent vertices of $v_{i}$ in $P_{n}$ passing through $v_{i}$ and it contributes a betweenness centrality 1 to $v_{i}$. Moreover, each pair $\left(v_{i-2}, v_{i+1}\right)$ and $\left(v_{i-1}, v_{i+2}\right)$ have two geodesic paths of length 3 , one of which passes through $v_{i}$. These two pairs provide a total of 1 to the centrality of $v_{i}$.

As a consequence, we have

$C_{B}\left(v_{i}\right)=\operatorname{deg}\left(v_{i}\right)-1+1+1=4$ for $i \in\{3,4, \ldots, n-2\}$, 
in which $\operatorname{deg}\left(v_{i}\right)=3$.

Case 2. Let $\bar{v}_{i}$ be a vertex in $\bar{P}_{n}$, where $i \in\{1,2, \ldots, n\}$. For all $i \geqslant 1$, consider the pair $\left(v_{j}, \bar{v}_{k}\right)$, where $j, k \in\{1,2, \ldots, n\}$ and $k \neq i$. There is only one geodesic path joining $v_{i}$ and adjacent vertices of $\bar{v}_{i}$ in $\bar{P}_{n}$ and it passes through $\bar{v}_{i}$. Since $\bar{v}_{i}$ has $\operatorname{deg}\left(\bar{v}_{i}\right)-1$ adjacent vertices in $\bar{P}_{n}$, it provides the betweenness centrality $\operatorname{deg}\left(\bar{v}_{i}\right)-1$ to $\bar{v}_{i}$.

We partitioned vertex set of $\bar{P}_{n}$ into three pairs for contribution of any pair of vertices in $P_{n}$ and similarly any pair of vertices in $\bar{P}_{n}$ to the betweenness centrality of $\bar{v}_{i}$.

- Let $i \in\{1,2,3\}$.

Consider pairs of vertices in $\bar{P}_{n}$. Now, for each pair $\left(\bar{v}_{j+2}, \bar{v}_{j+3}\right)$, where $j \in\{i, i+1, i+2, \ldots, n-3\}$, there is one geodesic path passing through $\bar{v}_{i}$. There are $n-i-2$ such pairs. While there are $n-3$ geodesic paths for the pair $\left(\bar{v}_{n-1}, \bar{v}_{n}\right)$, there are $n-4$ geodesic paths for the remaining $n-i-3$ pairs. Thus, these $n-i-2$ pairs contribute $\frac{1}{n-3}+\frac{n-i-3}{n-4}$ to the betweenness centrality of $\bar{v}_{i}$.

Consider pairs of vertices in $P_{n}$. If the distance between two vertices of $P_{n}$ is 1 or 2 in $P_{n}$, there is a geodesic path joining these vertices but it does not contain $\bar{v}_{i}$. Thus, consider paths of length at least 3 in $P_{n}$. There are two geodesic paths of length 3 joining $v_{i}$ and $v_{i+3}$, one of which passes through $\bar{v}_{i}$. Thereby, they contribute $\frac{1}{2}$ to the centrality of $\bar{v}_{i}$. Now, consider paths of length greater than 3 in $P_{n}$. Each pair $\left(v_{i}, v_{i+3+k}\right)$ has only one geodesic path and it passes through $\bar{v}_{i}$ for each $k \in\{1,2, \ldots, n-i-3\}$. Since there are $n-i-3$ such pairs, it contributes a betweenness centrality $n-i-3$ to $\bar{v}_{i}$.

As a consequence, for $i \in\{1,2,3\}$

$$
C_{B}\left(\bar{v}_{i}\right)=\operatorname{deg}\left(\bar{v}_{i}\right)+\frac{1}{n-3}+\frac{n-i-3}{n-4}+n-i-\frac{7}{2} .
$$

- Let $i \in\{4,5, \ldots, n-3\}$.

Consider the pair $\left(\bar{v}_{j+2}, \bar{v}_{j+3}\right)$, where $j \in$ $\{-1,0,1, \ldots, i-5, i, i+1, \ldots, n-3\}$. There are $n-5$ such pairs and two of them are $\left(\bar{v}_{1}, \bar{v}_{2}\right)$ and $\left(\bar{v}_{n-1}, \bar{v}_{n}\right)$. For these two pairs, there are $n-3$ geodesic paths of length 2 , one of them passes through $\bar{v}_{i}$. Hence, they contribute centrality $\frac{2}{n-3}$ to $\bar{v}_{i}$. For the remaining $n-7$ pairs, there are $n-4$ geodesic paths of length 2 , one of them passes through $\bar{v}_{i}$. Hence, each pair contributes centrality $\frac{1}{n-4}$ to $\bar{v}_{i}$ and they contribute a total of $\frac{n-7}{n-4}$. Therefore, $n-5$ pairs of vertices in $\bar{P}_{n}$ provides a betweenness centrality $\frac{2}{n-3}+\frac{n-7}{n-4}$ to $\bar{v}_{i}$.

Consider pairs of vertices in $P_{n}$. There is a geodesic path of length at least 3 passing through $\bar{v}_{i}$. Thus, we consider pairs $\left(v_{i-3-k}, v_{i}\right)$ and $\left(v_{i}, v_{i+3+l}\right)$, where $k \in\{0,1, \ldots, i-4\}$ and $l \in\{0,1, \ldots, n-i-3\}$. For each pair $\left(v_{i-3}, v_{i}\right)$ and $\left(v_{i}, v_{i+3}\right)$ where $k=0$ and $l=0$, there are two geodesic paths of length 3 and one of them passes through $\bar{v}_{i}$. Thus, they contribute a betweenness centrality
1 to $\bar{v}_{i}$. If $k>0$ or $l>0$, then each pair has only one geodesic path of length greater than 3 passing through $\bar{v}_{i}$. Since there are $n-7$ such pairs, they contribute a betweenness centrality $n-7$ to $\bar{v}_{i}$.

As a consequence, for $i \in\{4,5, \ldots, n-3\}$

$$
C_{B}\left(\bar{v}_{i}\right)=\operatorname{deg}\left(\bar{v}_{i}\right)+\frac{2}{n-3}+\frac{n-7}{n-4}+n-7 .
$$

- Let $i \in\{n-2, n-1, n\}$.

Consider the pair $\left(\bar{v}_{j}, \bar{v}_{j+1}\right)$ for $j \in\{1,2, \ldots, i-3\}$. There are $i-3$ such pairs and one of them is $\left(\bar{v}_{1}, \bar{v}_{2}\right)$. For this pair, there is $n-3$ geodesic paths joining $\bar{v}_{1}$ and $\bar{v}_{2}$, one of which passes through $\bar{v}_{i}$. Thus, it contributes $\frac{1}{n-3}$ to $\bar{v}_{i}$. For the remaining $i-4$ pairs, there are $n-4$ geodesic paths joining $\bar{v}_{j}$ and $\bar{v}_{j+1}$, one of which passes through $\bar{v}_{i}$ for each $j \in\{2,3, \ldots, i-3\}$. Each pair contributes $\frac{1}{n-4}$ to $\bar{v}_{i}$ giving a total of $\frac{i-4}{n-4}$.

Consider pairs of vertices in $P_{n}$. Among the paths of length 3 in $P_{n}$, there are two geodesic paths joining $v_{i}$ and $v_{i-3}$, one of which passes through $\bar{v}_{i}$. Hence, they contribute $\frac{1}{2}$ to $\bar{v}_{i}$. Now, consider all paths of length greater than 3 in $P_{n}$. There are $i-4$ geodesic paths joining $v_{i}$ and $v_{i-3-k}$, one of which passes through $\bar{v}_{i}$ for $k \in\{1,2, \ldots, i-4\}$. Thus, each pairs contributes centrality 1 to $\bar{v}_{i}$ giving a total of $i-4$.

As a consequence, for $i \in\{n-2, n-1, n\}$

$$
C_{B}\left(\bar{v}_{i}\right)=\operatorname{deg}\left(\bar{v}_{i}\right)+\frac{1}{n-3}+\frac{i-4}{n-4}-\frac{9}{2}+i .
$$

It is clear that $\operatorname{deg}\left(\bar{v}_{1}\right)=\operatorname{deg}\left(\bar{v}_{n}\right)=n-1$ and $\operatorname{deg}\left(\bar{v}_{i}\right)=$ $n-2$ for $i \neq 1, n$. Simplifying the equations of Case 2 ,

$$
C_{B}\left(\bar{v}_{i}\right)= \begin{cases}2 n+\frac{1}{n-3}-\frac{9}{2}, & \text { if } i \in\{1, n\}, \\ 2 n+\frac{1}{n-3}+\frac{n-5}{n-4}-\frac{15}{2}, & \text { if } i \in\{2, n-1\}, \\ 2 n+\frac{1}{n-3}+\frac{n-6}{n-4}-\frac{17}{2}, & \text { if } i \in\{3, n-2\}, \\ 2 n+\frac{2}{n-3}+\frac{n-7}{n-4}-9, & \text { if } i \in\{4,5, \ldots, n-3\} .\end{cases}
$$

is obtained.

The maximum centrality value of Case 2 is at the first or end vertex of $\bar{P}_{n}$. Comparing the maximum values of Case 1 and Case 2 we have

$$
C_{B}\left(v^{*}\right)=2 n+\frac{1}{n-3}-\frac{9}{2} .
$$

Thus, the betweenness centrality of $P_{n} \bar{P}_{n}$ is as follows:

$$
\begin{aligned}
C_{B}\left(P_{n} \bar{P}_{n}\right) & =\frac{2}{(2 n-1)^{2}(2 n-2)} \sum_{i=1}^{2 n}\left[C_{B}\left(v^{*}\right)-C_{B}\left(v_{i}\right)\right] \\
& =\frac{2 n^{3}-11 n^{2}+14 n+9}{(2 n-1)^{2}(n-1)(n-3)} .
\end{aligned}
$$

Theorem 2.6. The betweenness centrality of any vertex $v$ in the complementary prism of a cycle with $n>6$ is

$$
C_{B}(v)= \begin{cases}4, & \text { if } v \in V\left(C_{n}\right) \\ 2 n-8, & \text { if } v \in V\left(\bar{C}_{n}\right) .\end{cases}
$$


Proof. Since $\operatorname{diam}\left(C_{n} \bar{C}_{n}\right)=3$, there is a geodesic path of length at most 3 between two vertices in $C_{n} \bar{C}_{n}$. Since $C_{n}$ and $\bar{C}_{n}$ are vertex transitive, it is sufficient to consider without loss of generality that the betweenness centrality of any vertex in $C_{n}$ and similarly in $\bar{C}_{n}$.

Case 1. Let $v_{i}$ be any vertex in $C_{n}$, where $i \in\{1,2, \ldots, n\}$. The distance between non-adjacent vertices of $\bar{C}_{n}$ is 2 in $C_{n} \bar{C}_{n}$ and these vertices do not lie on any geodesic paths containing $v_{i}$. Then their betweenness centralities to $v_{i}$ are zero.

Consider pairs of vertices in $C_{n}$. For each pairs $\left(v_{i-1}, v_{i+2}\right)$ and $\left(v_{i-2}, v_{i+1}\right)$, there are two geodesic paths of length 3 , one of which passes through $v_{i}$. Note that, we take $v_{0}=v_{n}$ and $v_{-1}=v_{n-1}$. Among the paths of length less than 3 in $C_{n}$, there is only one geodesic path joining two adjacent vertices of $v_{i}$ in $C_{n}$ passes through $v_{i}$. Thus, they provide a total of 2 to the centrality of $v_{i}$.

Now, consider the paths joining vertices of $C_{n}$ and vertices of $\bar{C}_{n}$. There is only one geodesic path joining $\bar{v}_{i}$ and two adjacent vertices of $v_{i}$ in $C_{n}$ passing through $v_{i}$. Thus, each one contributes 1 to the centrality of $v_{i}$ and they give a total of 2 .

Consequently, the betweenness centrality of any vertex in $C_{n}$ is 4 .

Case 2. Let $\bar{v}_{i}$ be any vertex in $\bar{C}_{n}$, where $i \in\{1,2, \ldots, n\}$. Consider pairs of vertices in $C_{n}$. There are two vertices in $C_{n}$ at distance 3 with $v_{i}$, and there are two geodesic paths joining $v_{i}$ and each of these two vertices. One of two geodesic paths passes through $\bar{v}_{i}$. Thus, each one contributes $\frac{1}{2}$ to the centrality of $\bar{v}_{i}$ and they give a total of 1. Furthermore, each geodesic path joining $v_{i}$ and $n-7$ vertices of $C_{n}$ that the distance with $v_{i}$ in $C_{n}$ is greater than 3 , if any, contributes 1 to the centrality of $\bar{v}_{i}$. Then it gives a total of $n-7$.

Consider pairs of vertices between $V\left(C_{n}\right)$ and $V\left(\bar{C}_{n}\right)$. There is only one geodesic path joining vertex $v_{i}$ and each adjacent vertex of $\bar{v}_{i}$ in $\bar{C}_{n}$. Since there are $n-3$ such adjacent vertices, they contribute a betweenness centrality $n-3$ to $\bar{v}_{i}$.

Consider pairs of vertices in $\bar{C}_{n}$. Since there are $n-3$ adjacent vertices of $\bar{v}_{i}$ in $\bar{C}_{n}$, there are $n-4$ non-adjacent pairs of vertices with these $n-3$ vertices. For each pair, there are $n-4$ geodesic paths joining its vertices, one of which passes through $\bar{v}_{i}$. Thus, $n-4$ pairs contribute 1 to the centrality of $\bar{v}_{i}$.

Consequently, the betweenness centrality of any vertex in $\bar{C}_{n}$ is $2 n-8$.

The largest value of the betweenness centrality of vertices of $C_{n} \bar{C}_{n}$

$$
C_{B}\left(v^{*}\right)=2 n-8,
$$

and the betweenness centrality of $C_{n} \bar{C}_{n}$ is

$$
C_{B}\left(C_{n} \bar{C}_{n}\right)=\frac{2 \sum_{i=1}^{2 n}\left[C_{B}\left(v^{*}\right)-C_{B}\left(v_{i}\right)\right]}{(2 n-1)^{2}(2 n-2)}=\frac{2 n(n-6)}{(2 n-1)^{2}(n-1)} .
$$

Theorem 2.7. Let $K_{n, m} \bar{K}_{n, m}$ be the complementary prism of a complete bipartite graph with $n \leqslant m$. Then the betweenness centrality of a vertex $v$ in $K_{n, m} \bar{K}_{n, m}$ is given by

$$
C_{B}(v)= \begin{cases}\frac{m^{2}-m+4 m n}{2 n}, & \text { if } \operatorname{deg}(v)=m+1, \\ \frac{n^{2}-n+4 m n}{2 m}, & \text { if } \operatorname{deg}(v)=n+1, \\ n-1, & \text { if } \operatorname{deg}(v)=n, \\ m-1, & \text { if } \operatorname{deg}(v)=m .\end{cases}
$$

Proof. Let $V\left(K_{n, m} \bar{K}_{n, m}\right)=V_{1}^{(1)} \cup V_{1}^{(2)} \cup V_{2}^{(1)} \cup V_{2}^{(2)}$, where $V_{1}^{(1)}=\left\{v_{1}, v_{2}, \ldots, v_{n}\right\}, V_{1}^{(2)}=\left\{v_{n+1}, v_{n+2}, \ldots, v_{n+m}\right\}$ and $V_{2}^{(1)}=\left\{\bar{v}_{1}, \bar{v}_{2}, \ldots, \bar{v}_{n}\right\}, V_{2}^{(2)}=\left\{\bar{v}_{n+1}, \bar{v}_{n+2}, \ldots, \bar{v}_{n+m}\right\}$. The degree of any vertex of $V_{1}^{(1)}, V_{1}^{(2)}, V_{2}^{(1)}$ and $V_{2}^{(2)}$ in $K_{n, m} \bar{K}_{n, m}$ are $m+1, n+1, n$ and $m$, respectively. Then we have following cases:

Case 1. Let $v_{i}$ be a vertex in $V_{1}^{(1)}$, where $i \in\{1,2, \ldots, n\}$. Consider pairs of vertices $\left(v_{j}, v_{k}\right)$, where $j, k \in\{n+1, n+2, \ldots, n+m\}$ and $j \neq k$. For each pair, there are $n$ geodesic paths joining the adjacent vertices of $v_{j}$ in $V_{1}^{(1)}$ and $v_{k}$, one of which passes through $v_{i}$. Thus, each pair contributes centrality $\frac{1}{n}$ to $v_{i}$ and they give a total of $\left(\begin{array}{c}m \\ 2\end{array}\right) \frac{1}{n}$.

Consider pairs of vertices $\left(v_{j}, \bar{v}_{k}\right)$ for $v_{j} \in V_{1}^{(2)}$ and $\bar{v}_{k} \in V_{2}^{(1)}$. For these pairs there is only one geodesic path joining vertex $v_{j}$ and corresponding vertex of $v_{i}$ in $V_{2}^{(1)}$ containing $v_{i}$. Since there are $m$ such pairs, they contribute a betweenness centrality $m$ to $v_{i}$.

Consider pairs of vertices between $V_{2}^{(1)}$ and $V_{2}^{(2)}$. There is only one geodesic path joining corresponding vertex of $v_{i}$ in $V_{2}^{(1)}$ and any vertex of $V_{2}^{(2)}$ passing through $v_{i}$. Since $\left|V_{2}^{(2)}\right|=m$, this contributes centrality $m$ to $v_{i}$. The remaining pairs of vertices do not lie on any geodesic paths passing through $v_{i}$. Thus, their contribution of the betweenness centrality to $v_{i}$ is zero.

As a consequence, the betweenness centrality of $v_{i}$ in $V_{1}^{(1)}$ is

$$
\left(\begin{array}{c}
m \\
2
\end{array}\right) \frac{1}{n}+2 m=\frac{m^{2}-m+4 m n}{2 n} .
$$

Case 2. Let $v_{i}$ be a vertex in $V_{1}^{(2)}$, where $i \in\{n+1, n+$ $2, \ldots, n+m\}$. The proof is similar to that of Case 1 and is omitted. Hence, we have

$$
C_{B}\left(v_{i}\right)=\frac{n^{2}-n+4 m n}{2 m} .
$$

Case 3. Let $\bar{v}_{i}$ be a vertex in $V_{2}^{(1)}$, where $i \in\{1,2, \ldots, n\}$. In this case, there is only one geodesic path from 
corresponding vertex of $\bar{v}_{i}$ in $V_{1}^{(1)}$ to any vertex $\bar{v}_{k}$ passing through $\bar{v}_{i}$ for each $k \in\{1,2, \ldots, n\}$ and $k \neq i$. Since there are $n-1$ such pairs, they contribute $n-1$ to $\bar{v}_{i}$. None of other pairs lies on any geodesic paths. As a consequence, the betweenness centrality of any vertex $\bar{v}_{i}$ in $V_{2}^{(1)}$ is $n-1$.

Case 4. Let $\bar{v}_{i}$ be a vertex in $V_{2}^{(2)}$, where $i \in\{n+1, n+2, \ldots, n+m\}$.

When this case is proved similar to Case 3 , we have $C_{B}\left(v_{i}\right)=m-1$.

The largest value of the betweenness centrality of vertices of $K_{n, m} \bar{K}_{n, m}$ is

$$
C_{B}\left(v^{*}\right)=\frac{m^{2}-m+4 m n}{2 n},
$$

and the betweenness centrality of $K_{n, m} \bar{K}_{n, m}$ is

$$
\begin{aligned}
C_{B}\left(K_{n, m} \bar{K}_{n, m}\right) & =\frac{2 \sum_{i=1}^{2(n+m)}\left[C_{B}\left(v^{*}\right)-C_{B}\left(v_{i}\right)\right]}{(2(n+m)-1)^{2}(2(n+m)-2)} \\
& =\frac{m n+3 n^{2}+7 m^{2} n-3 n^{3}+m^{2}(2 m-2)}{2 n(2 n+2 m-1)^{2}(n+m-1)} .
\end{aligned}
$$

Theorem 2.8. For the complementary prism of a wheel graph $W_{1, n} \bar{W}_{1, n}$ of order $2 n+2$, let c and $\bar{c}$ be center vertex of $W_{1, n}$ and corresponding vertex of $c$ in $\bar{W}_{1, n}$, respectively. Then the betweenness centrality of a vertex $v$ in $W_{1, n} \bar{W}_{1, n}$ for $n>5$ is given by

$$
C_{B}(v)= \begin{cases}\frac{n^{2}}{2}, & \text { if } v=c \\ 0, & \text { if } v=\bar{c} \\ \frac{9}{2}, & \text { if } v \text { is in } W_{1, n}-\{c\} \\ n-2, & \text { if } v \text { is in } \bar{W}_{1, n}-\{\bar{c}\}\end{cases}
$$

Proof.Let $V\left(W_{1, n}\right)=\left\{c, v_{1}, v_{2}, \ldots, v_{n}\right\}$ and $V\left(\bar{W}_{1, n}\right)=$ $\left\{\bar{c}, \bar{v}_{1}, \bar{v}_{2}, \ldots, \bar{v}_{n}\right\}$, where $c$ is center vertex of $W_{1, n}$ and $\bar{c}$ is corresponding vertex of $c$ in $\bar{W}_{1, n}$. Wheel graph $W_{1, n}$ contains a cycle of order $n$ and center vertex is adjacent to each vertex of cycle $C_{n}$. Then we have following four cases:

Case 1. Take center vertex $c$ of $W_{1, n}$.

Since $\operatorname{diam}\left(W_{1, n}\right)=2$, the distance between two vertices of $C_{n}$ in $W_{1, n} \bar{W}_{1, n}$ is at most 2. Thus, by Theorem 2.2 vertices of $W_{1, n}$ in $W_{1, n} \bar{W}_{1, n}$ contribute a betweenness centrality $\frac{n(n-4)}{2}$ to $c$.

Among the paths between any vertex of $W_{1, n}$ and any vertex of $\bar{W}_{1, n}$, there is only one geodesic path of length 2 joining $\bar{c}$ and $v_{i}$ for each $i \in\{1,2, \ldots, n\}$ passing through $c$. Thus, each path contributes 1 to the centrality of $c$ and they give a total of $n$.

Consider pairs of vertices in $\bar{W}_{1, n}$. Since the distance between any two vertices in $\bar{W}_{1, n}-\{\bar{c}\}$ is at most 2 in $W_{1, n} \bar{W}_{1, n}$, none of paths joining two vertices of $\bar{W}_{1, n}-\{\bar{c}\}$ contains $c$. Only there is one geodesic path joining vertex $\bar{c}$ and vertex $\bar{v}_{i}$ passing through $c$ for each $i \in\{1,2, \ldots, n\}$.
Since there is $n$ such pairs, they contribute centrality $n$ to $c$.

As a consequence, the betweenness centrality of $c$ is $\frac{n(n-4)}{2}+2 n=\frac{n^{2}}{2}$.

Case 2. Take corresponding vertex of center vertex. Since $\operatorname{deg}(\bar{c})=1$, there is one path joining $\bar{c}$ and all other vertices. However, no one contains $\bar{c}$. Thus, the betweenness centrality of $\bar{c}$ is zero.

Case 3. Consider any vertex $v_{i}$ in $W_{1, n}-\{c\}$, where $i \in\{1,2, \ldots, n\}$.

There are two geodesic paths joining adjacent vertices of $v_{i}$ on $C_{n}$, one of them passes through $v_{i}$. Thus, its contribution to the centrality of $v_{i}$ is $\frac{1}{2}$.

After examining the geodesic paths joining vertices of $W_{1, n}$ and vertices of $\bar{W}_{1, n}$, it is seen that there is a geodesic path of length 2 only joining corresponding vertex of $v_{i}$ and adjacent vertex of $v_{i}$ in $W_{1, n}$ passing through $v_{i}$. Since $v_{i}$ has three adjacent vertices in $W_{1, n}$, they contribute a betweenness centrality 3 to $v_{i}$.

Now, consider pairs of vertices in $\bar{W}_{1, n}$. Since the distance between any two vertices of $\bar{W}_{1, n}-\{\bar{c}\}$ is at most 2 in $W_{1, n} \bar{W}_{1, n}$, no paths joining vertices of $\bar{W}_{1, n}-\{\bar{c}\}$ pass through $v_{i}$. However, there is a geodesic path of length 3 joining vertex $\bar{c}$ and vertex $\bar{v}_{i}$ passing through $v_{i}$. It contributes a betweenness centrality 1 to $v_{i}$.

As a consequence, the betweenness centrality of any vertex in $W_{1, n}-\{c\}$ is $\frac{1}{2}+3+1=\frac{9}{2}$.

Case 4. Consider any vertex $\bar{v}_{i}$ in $\bar{W}_{1, n}-\{\bar{c}\}$, where $i \in\{1,2, \ldots, n\}$.

There is a geodesic path joining $v_{i}$ and adjacent vertices of $\bar{v}_{i}$ in $\bar{W}_{1, n}$ and it contains $\bar{v}_{i}$. Since $\bar{v}_{i}$ has $n-3$ adjacent vertices in $\bar{W}_{1, n}$, their contribution is $n-3$ to $v_{i}$. The distance between all pair of vertices of $W_{1, n}$ is at most 2 in $W_{1, n} \bar{W}_{1, n}$. Thus, none of paths joining these vertices contains $\bar{v}_{i}$.

Among pairs of vertices in $\bar{W}_{1, n}$, consider the pair $\left(\bar{v}_{j(\bmod n)}, \bar{v}_{j+1(\bmod n)}\right)$, where $j \in\{i+2, i+3, \ldots, n+i-3\}$ and $\bar{v}_{0}=\bar{v}_{n}$. There are $n-4$ geodesic paths joining $\bar{v}_{j}$ and $\bar{v}_{j+1}$ for each $j \in\{i+2, i+3, \ldots, n+i-3\}$ and one of these contains $\bar{v}_{i}$. Since there are $n-4$ such pairs, they contribute a betweenness centrality 1 to $\bar{v}_{i}$.

As a consequence, the betweenness centrality of any vertex in $\bar{W}_{1, n}-\{\bar{c}\}$ is $n-3+1=n-2$.

The largest value $C_{B}\left(v^{*}\right)$ is $\frac{n^{2}}{2}$ and the graph centrality is as follows:

$C_{B}\left(W_{1, n} \bar{W}_{1, n}\right)=\frac{2 \sum_{i=1}^{2(n+1)}\left[C_{B}\left(v^{*}\right)-C_{B}\left(v_{i}\right)\right]}{2 n(2 n+1)^{2}}=\frac{2 n^{2}-n-5}{2(2 n+1)^{2}}$. 


\section{References}

[1] Bader, D. A., Kintali, S., Madduri, K., Mihail, M. 2007. Approximating Betweenness Centrality. In International Workshop on Algorithms and Models for the Web-Graph, 124-137. Springer Berlin Heidelberg.

[2] Raghavan Unnithan, S. K., Kannan, B., Jathavedan, M. 2014. Betweenness Centrality in Some Classes of Graphs. International Journal of Combinatorics, 2014, Article ID 241723, 12 pages.

[3] Otte, E., Rousseau, R. 2002. Social Network Analysis: a powerful strategy, also for the information sciences. Journal of information Science, 28(6), 441-453.

[4] Latora, V., Marchiori, M. 2007. A Measure of Centrality Based on Network Efficiency. New Journal of Physics, 9(6), 188.

[5] Estrada, E. 2006. Virtual Identification of Essential Proteins within the Protein Interaction Network of Yeast. Proteomics, 6(1), 35-40.

[6] Rubinov, M., Sporns, O. 2010. Complex Network Measures of Brain Connectivity: uses and interpretations. Neuroimage, 52(3), 1059-1069.

[7] Dehmer, M., Emmert-Streib, F. (Eds.). 2014. Quantitative Graph Theory: Mathematical Foundations and Applications. CRC press, 516p.

[8] Bavelas, A. 1948. A Mathematical Model for Group Structures. Human organization. 7(3), 16-30.
[9] Freeman, L. C. 1977. A Set of Measures of Centrality Based on Betweenness. Sociometry, 40(1), 35-41.

[10] Haynes, T. W., Henning, M. A., Slater, P. J., van der Merwe, L. C. 2007. The Complementary Product of Two Graphs. Bulletin of the Institute of Combinatorics and its Applications, 51, 21-30.

[11] Cappelle, M. R., Coelho, E. M., Coelho, H., Penso, L. D., Rautenbach, D. 2015. Identifying Codes in the Complementary Prism of Cycles. arXiv preprint arXiv:1507.05083.

[12] Haynes, T. W., Henning, M. A., van der Merwe, L. C. 2009. Domination and Total Domination in Complementary Prisms. Journal of Combinatorial Optimization, 18(1), 23-37.

[13] Chaluvaraju, B., Chaitra, V. 2012. Roman domination in complementary prism graphs. Mathematical Combinatorics, 2, 24-31.

[14] Desormeaux, W. J., Haynes, T. W., Vaughan, L. 2013. Double domination in complementary prisms. Utilitas Mathematica, 91, 131-142.

[15] Kazemi, A. P. 2012. k-Tuple Total Domination in Complementary Prisms. ISRN Discrete Mathematics, 2011.

[16] Chartrand, G., Lesniak, L., Zhang, P. 2010. Graphs \& Digraphs, Fifth Edition. Taylor \& Francis. CRC Press. $586 \mathrm{p}$. 